\title{
A micro electromagnetic generator for vibration energy harvesting
}

\author{
S P Beeby ${ }^{1}$, R N Torah ${ }^{1}$, M J Tudor ${ }^{1}$, P Glynne-Jones ${ }^{1}$, \\ T O'Donnell ${ }^{2}$, C R Saha ${ }^{2}$ and S Roy ${ }^{2}$ \\ ${ }^{1}$ School of Electronics and Computer Science, University of Southampton, Highfield, \\ Southampton, Hampshire, SO17 1BJ, UK \\ ${ }^{2}$ Tyndall National Institute, Prospect Row, Cork, Republic of Ireland \\ E-mail: spb@ecs.soton.ac.uk
}

Received 22 March 2007, in final form 6 May 2007

Published 5 June 2007

Online at stacks.iop.org/JMM/17/1257

\begin{abstract}
Vibration energy harvesting is receiving a considerable amount of interest as a means for powering wireless sensor nodes. This paper presents a small (component volume $0.1 \mathrm{~cm}^{3}$, practical volume $0.15 \mathrm{~cm}^{3}$ ) electromagnetic generator utilizing discrete components and optimized for a low ambient vibration level based upon real application data. The generator uses four magnets arranged on an etched cantilever with a wound coil located within the moving magnetic field. Magnet size and coil properties were optimized, with the final device producing $46 \mu \mathrm{W}$ in a resistive load of $4 \mathrm{k} \Omega$ from just $0.59 \mathrm{~m} \mathrm{~s}^{-2}$ acceleration levels at its resonant frequency of $52 \mathrm{~Hz}$. A voltage of $428 \mathrm{mVrms}$ was obtained from the generator with a 2300 turn coil which has proved sufficient for subsequent rectification and voltage step-up circuitry. The generator delivers $30 \%$ of the power supplied from the environment to useful electrical power in the load. This generator compares very favourably with other demonstrated examples in the literature, both in terms of normalized power density and efficiency.
\end{abstract}

(Some figures in this article are in colour only in the electronic version)

\section{Introduction}

Wireless sensor systems are receiving increasing interest since they offer flexibility, ease of implementation and the ability to retrofit systems without the cost and inconvenience of cabling. Furthermore, by removing wires there is the potential for embedding sensors in previously inaccessible locations. At present, the majority of wireless sensor nodes are simply battery-powered. Despite measures such as low power techniques for communicating (e.g. IEEE 802.15.4 and Zigbee protocols) and the intelligent management of the sensor node's power consumption, batteries will still require periodical replacement. Replacing batteries is not compatible with embedded applications nor is it feasible for networks with large numbers of nodes.

The advances made in low power wireless systems present an opportunity for alternative types of power source. Solutions such as micro fuel cells [1] and micro turbine generators [2] are capable of high levels of energy and power density.
However, they involve the use of chemical energy and require refuelling. Energy harvesting approaches that transform light, heat and kinetic energy available in the sensor's environment into electrical energy offer the potential of renewable power sources which can be used to directly replace or augment the battery. Such renewable sources could increase the lifetime and capability of the network and mitigate the environmental impact caused by the disposal of batteries. In this context, solar power is the most well known.

The subject of this paper is a kinetic energy generator which converts mechanical energy in the form of vibrations present in the application environment into electrical energy. Kinetic energy is typically converted into electrical energy using electromagnetic, piezoelectric or electrostatic transduction mechanisms [3]. Vibrations are an attractive source since the energy present can be harvested by compact inertial devices that benefit from a high $Q$-factor amplifying the base excitation amplitude. Suitable vibrations can be found in numerous applications including common household goods 


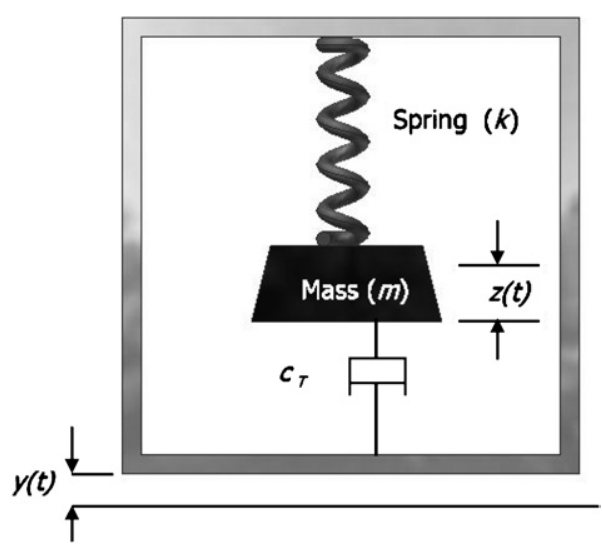

Figure 1. Model of a linear, inertial generator.

(fridges, washing machines, microwave ovens), industrial plant equipment, moving vehicles such as automobiles and aeroplanes and structures such as buildings and bridges [4]. Human-based applications are characterized by low frequency high amplitude displacements $[5,6]$.

The amount of energy generated by this approach fundamentally depends upon the quantity and form of the kinetic energy available in the application environment and the efficiency of the generator and the power conversion electronics. This paper presents the development of an electromagnetic micro generator designed to harvest energy from the vibrations of an air compressor unit which exhibits large vibration maxima in the range of $0.19-3.7 \mathrm{~m} \mathrm{~s}^{-2}$ at frequencies between $43 \mathrm{~Hz}$ and $109 \mathrm{~Hz}$. The micro generator was therefore designed to operate within this range and to be as small as possible whilst still generating useable levels of power and voltage. The paper presents a theoretical analysis of inertial generators, the design, simulation and testing of the electromagnetic generator and a comparison with other inertial generators. This work was carried out as part of the European Union funded project 'Vibration Energy Scavenging' (VIBES).

\section{Basic theory}

Resonant generators can be modelled as a second-order, spring-mass-damper system with base excitation. Figure 1 shows a general example of such a system based on a seismic mass, $m$, on a spring of stiffness, $k$. Total energy losses within the system are represented by the damping coefficient, $c_{T}$. These losses consist of parasitic loss mechanisms (e.g. air damping), represented by $c_{p}$, and electrical energy extracted by the transduction mechanism, represented by $c_{e}$.

These generators are intended to operate at their resonant frequency and for optimum energy extraction should be designed such that this coincides with the vibrations present in the intended application environment. The theory of inertialbased generators is well documented [7-9] and will only be briefly summarized here. Assuming the generator is driven by a harmonic base excitation $y(t)=Y \sin (\omega t)$, it will move out of phase with the mass at resonance resulting in a net displacement, $z(t)$, between the mass and the frame.
The average power dissipated within the damper (i.e. the power extracted by the transduction mechanism and the power lost through parasitic damping mechanisms) is given by:

$$
P_{\mathrm{av}}=\frac{m \xi_{T} Y^{2}\left(\frac{\omega}{\omega_{n}}\right)^{3} \omega^{3}}{\left[1-\left(\frac{\omega}{\omega_{n}}\right)^{2}\right]^{2}+\left[2 \xi_{T}\left(\frac{\omega}{\omega_{n}}\right)\right]^{2}}
$$

where $\xi_{T}$ is the total damping ratio given by $\xi_{T}=c_{T} / 2 m \omega_{n}$. Since this equation is valid for steady-state conditions, $P_{\mathrm{av}}$ is equal to the kinetic energy supplied per second by the application vibrations. Maximum power dissipation within the generator occurs when the device is operated at $\omega_{n}$ and in this case $P_{\text {av }}$ is given by:

$$
P_{\mathrm{av}}=\frac{m Y^{2} \omega_{n}^{3}}{4 \xi_{T}}
$$

Equation (2) suggests the following rules: (a) power varies linearly with the mass; (b) power increases with the cube of the frequency and (c) power increases with the square of the base amplitude. Rules (b) and (c) are dependant upon the base excitation, i.e. the accelerations present in the application environment. Since the peak acceleration of the base, $A$, is given by $A=\omega^{2} Y$ and damping factor is related to the damping ratio by $c_{T}=2 m \omega_{n} \xi_{T}$, equation (2) can also be written in the form

$$
P_{\mathrm{av}}=\frac{(m A)^{2}}{2 c_{T}} \text {. }
$$

These equations emphasize the need to understand the vibrations present in the intended application when designing an inertial generator. However, one cannot simply choose a particular frequency of operation based upon the power output alone. The inertial mass displacement will be limited to a given finite value, $z_{\max }$, depending upon the size of the generator, its design and material limitations. This is especially relevant in the case of MEMS generators. Furthermore, $z_{\max }$ will be a multiple $Q_{T}$ times larger than $Y$ where $Q_{T}$ is the total quality factor of the generator given by equation (4):

$$
Q_{T}=\frac{\omega_{n} m}{c_{T}}=\frac{1}{2 \xi_{T}} \text {. }
$$

The relationship between $Q_{T}$ and the electrical and parasitic damping factors is given by equation (5) where $Q_{\mathrm{OC}}$ is the open circuit $Q$-factor, i.e. $1 / 2 \xi_{P}$, and $Q_{E}$ is equal to $1 / 2 \xi_{E}$.

$$
\frac{1}{Q_{T}}=\frac{1}{Q_{\mathrm{OC}}}+\frac{1}{Q_{E}} \text {. }
$$

Taking $z_{\max }$ into consideration, average power can also be expressed as

$$
P_{\mathrm{av}}=\frac{m \omega_{n}^{3} Y z_{\max }}{2} .
$$

Incorporating the parasitic and electrical damping into equation (2) gives the average power delivered to the electrical domain:

$$
P_{\text {avelec }}=\frac{m \xi_{E} Y^{2} \omega_{n}^{3}}{4\left(\xi_{P}+\xi_{E}\right)^{2}} .
$$

Maximum power is delivered to electrical domain when $\xi_{E}=\xi_{P}$ i.e. damping arising from the electrical domain should equal mechanical losses. In this case equation (7) simplifies to

$$
P_{\text {avelec }}=\frac{m Y^{2} \omega_{n}^{3}}{16 \xi_{P}}
$$

Not all the energy transduced into the electrical domain will actually be delivered into the load. In the case of 

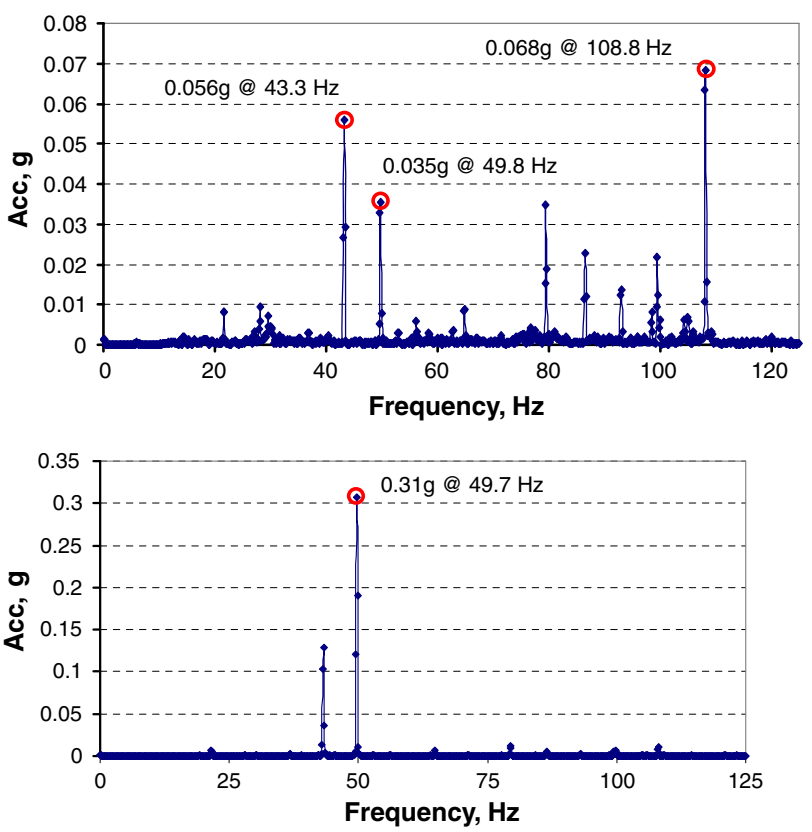

Figure 2. Example vibration spectra from compressor application (top plot from compressor enclosure, bottom plot from compressor).

electromagnetic transduction, some of the power delivered to the electrical domain is lost within the coil. The actual power in the load is a function of the coil and load resistances and is calculated from equation (9)

$$
P_{L \max }=\frac{m \omega_{n}^{3} Y^{2}}{16 \xi_{P}}\left(\frac{R_{\text {load }}}{R_{\text {load }}+R_{\text {coil }}}\right) .
$$

However, the coil and load resistances also affect the damping factor arising from electromagnetic transduction $c_{E}$ which can be estimated from equation (10) where $N$ is the number of turns in the generator coil, $l$ is the side length of the coil (assumed square) and $B$ is the flux density to which it is subjected. $R_{L}$, $R_{\text {coil }}$ and $L_{\text {coil }}$ are the load resistance, coil resistance and coil inductance respectively. Equation (12) is an approximation and only ideal for the case where the coil moves in a region of constant magnetic field.

$$
c_{E}=\frac{(N l B)^{2}}{R_{L}+R_{\text {coil }}+j \omega L_{\text {coil }}} .
$$

\section{Application overview}

The intended application for the generators described in this paper is an air compressor unit supplying several laboratories within a building. The electric motor runs continuously whilst the compressor is duty cycled to maintain the pressure within an in-line reservoir tank. The vibration levels and frequencies have been measured at various locations on the compressor and electric motor. The measured results indicate several resonances between 43 and $109 \mathrm{~Hz}$ with acceleration levels between 0.19 and $3.7 \mathrm{~m} \mathrm{~s}^{-2}$. Example vibration spectra taken from the side of the compressor enclosure and the top of the compressor are shown in figure 2. The generators presented in this paper have been designed to operate at these lower frequencies and at an rms acceleration of $0.59 \mathrm{~m} \mathrm{~s}^{-2}$ (or $60 \mathrm{~m} g$ where $1 \mathrm{~g}=9.81 \mathrm{~m} \mathrm{~s}^{-2}$ ). This frequency range and

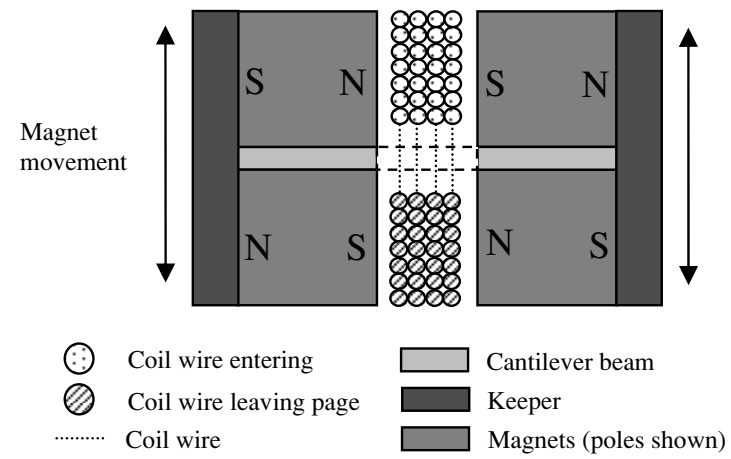

Figure 3. Cross section through the four-magnet arrangement.

acceleration level is indicative of the vibration levels found in typical industrial applications.

\section{Mk1 electromagnetic generator design}

\subsection{Generator design overview}

The micro electromagnetic generators presented in this paper are a miniaturized form of a previous larger scale design [10]. The generator uses miniature discrete components fabricated using a variety of conventional manufacturing processes. This enables the generator to exploit the advantages of bulk magnetic material properties and large coil winding density thereby demonstrating useable levels of power from a compact design. A comparison between bulk and integrated components for electromagnetic vibration energy harvesting has been presented elsewhere [11].

The design uses four high energy density sintered rare earth neodymium iron boron $(\mathrm{NdFeB})$ magnets manually bonded with Cyanoacrylate to the top and bottom surfaces of a cantilever beam with the aid of an alignment jig. The magnets were $1 \times 1 \times 1.5 \mathrm{~mm}^{3}$ in size, being $1.5 \mathrm{~mm}$ in the poled direction. The magnetic poles are aligned as shown in figure 3. The magnetic circuit is completed by zinc coated mild steel keepers which couple the flux between top and bottom magnets. This arrangement produces a concentrated flux gradient through the stationary coil as the magnets vibrate.

Additional mass is added to the generator in the form of two wire eroded tungsten alloy blocks attached to the free end of the cantilever beam. The tungsten alloy has a density of $18.1 \mathrm{~g} \mathrm{~cm}^{-3}$ providing a compact inertial mass. The density of the magnets is $7.6 \mathrm{~g} \mathrm{~cm}^{-3}$.

The beam used in this design was $9 \mathrm{~mm}$ long, $3 \mathrm{~mm}$ wide along $7 \mathrm{~mm}$ of the beam length and $4 \mathrm{~mm}$ wide for the final $2 \mathrm{~mm}$. Slots and holes have been incorporated into the beam to accommodate the coil and bolt. All corners have radii to reduce stress concentration effects. For the Mk1 generator, beams were fabricated from double polished single crystal silicon wafers. The geometry of the beam and required thickness was determined by finite element analysis. A thickness of $50 \mu \mathrm{m}$ gave resonant frequencies between 50 and $60 \mathrm{~Hz}$. Double polished wafers were purchased in the desired thickness (with a 5\% tolerance), therefore having a high quality finish on both top and bottom surfaces. The wafers were resist bonded to a host wafer and the beams fabricated by deep reactive ion etching through the $50 \mu \mathrm{m}$ thickness. 


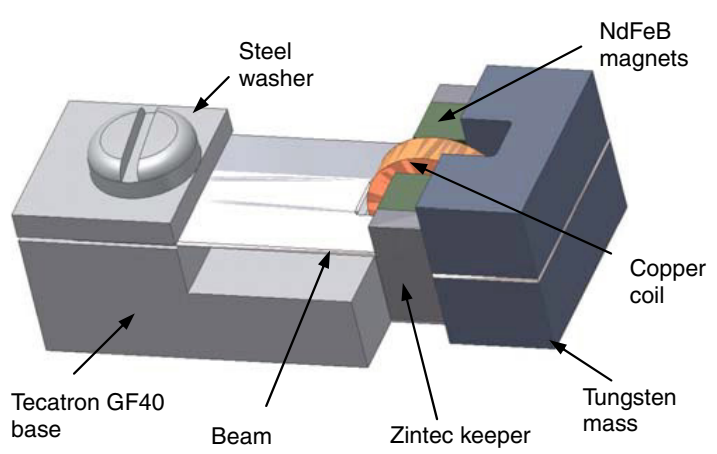

Figure 4. Micro cantilever generator.

The cantilever beam assembly was clamped onto the base using an M1 sized nut and bolt and a square washer. The square washer gives a straight clamped edge perpendicular to beam length. The base is machined from Tecatron GF40, a $40 \%$ glass fibre reinforced semi-crystalline high performance plastic using a Daytron micro-mill. The high rigidity of the material provides a firm clamping edge which is important to avoid excessive energy loss through the fixed end of the beam. The coil was manually bonded to a semi-circular recess machined in the base. The coil has an outside radius $R_{o}$ of $1.2 \mathrm{~mm}$, an inside radius $R_{i}$ of $0.3 \mathrm{~mm}$ and a thickness $t$ of $0.5 \mathrm{~mm}$. It was wound from $25 \mu \mathrm{m}$ diameter enamelled copper wire and had 600 turns. A drawing of the assembled generator is shown in figure 4. With the aid of alignment jigs, a tolerance of better than $0.1 \mathrm{~mm}$ can be achieved with the manual assembly of the components. The volume of the generator components is $0.1 \mathrm{~cm}^{3}$ whilst the practical volume, i.e. including the swept volume of the beam, is approximately $0.15 \mathrm{~cm}^{3}$.

\subsection{Mkl generator results}

The generator produced a peak power of $10.8 \mu \mathrm{W}$ from $60 \mathrm{mg}$ acceleration $\left(1 g=9.81 \mathrm{~m} \mathrm{~s}^{-2}\right)$ across a $110 \Omega$ load. The voltage level generated was $34.5 \mathrm{mVrms}$. The generator also demonstrated nonlinear behaviour which produced a significant level of hysteresis in the output. This is shown in figure 5 where the power output was measured as the frequency was increased from below to above resonance and also as the frequency was decreased from above to below resonance. When reducing frequency the maximum power that can be obtained is $2.5 \mu \mathrm{W}$. Whilst useable levels of power were delivered to the load, the voltage level was too low to enable subsequent voltage signal conditioning.

\section{MK2 electromagnetic generator design}

The generator was next subject to an optimization process with the objectives of increasing the generated voltage and power levels. In particular, the magnet size, beam material and coil parameters were investigated

\subsection{Finite element magnetic modelling}

Ansoft Maxwell 3D magnetic finite element (FE) software was used to optimize the electromagnetic circuit. The influence of

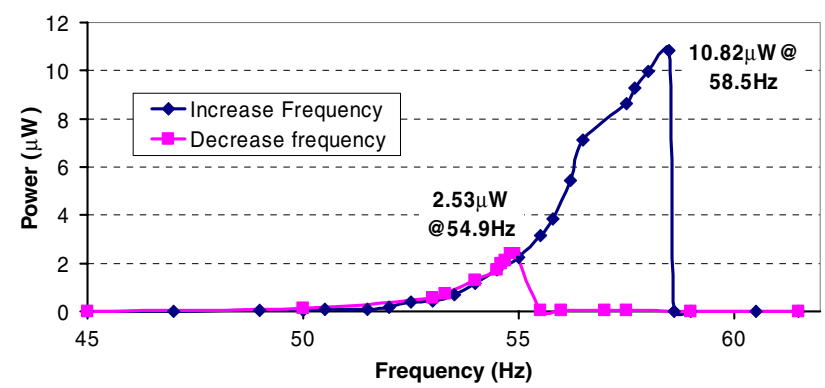

Figure 5. Power output hysteresis effect for the $50 \mu \mathrm{m}$ beam micro generator.

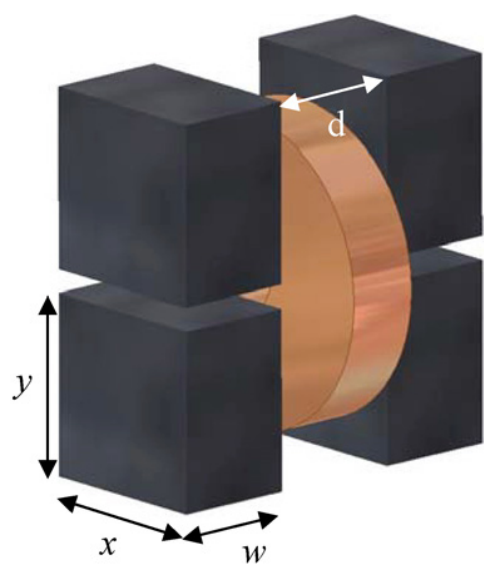

Figure 6. Magnet dimensions for simulation results.

magnet size was investigated by comparing the open circuit voltage for various magnet widths and heights (dimensions $x$ and $y$ respectively in figure 6). The thickness of the magnet, $w$, was fixed at $1.5 \mathrm{~mm}$ and the distance between the magnets, $d$, was fixed at $1 \mathrm{~mm}$. The simulations were carried out with an excitation frequency of $60 \mathrm{~Hz}$, and acceleration of $0.59 \mathrm{~m} \mathrm{~s}^{-2}$. Given a peak magnet amplitude of $0.57 \mathrm{~mm}$, this corresponds to a $Q$-factor of 140 .

First, dimension $y$ was fixed at $1 \mathrm{~mm}$ and $x$ was varied between 1 and $3 \mathrm{~mm}$. The peak-generated voltage rises with increasing $x$, but the rate of improvement reduces beyond $2.5 \mathrm{~mm}$. Since, for a given volume, increasing magnet width causes a reduction in the size of the proof mass, dimension $x$ was fixed at $2.5 \mathrm{~mm}$. Next, with $x$ fixed, $y$ was adjusted between 1 and $3 \mathrm{~mm}$. The simulation results again show an improvement in generated voltage with increasing $y$ up to $2 \mathrm{~mm}$. The simulation identified a practical optimum magnet size of $2.5 \times 2 \times 1.5 \mathrm{~mm}^{3}$ with further increases in magnet size yielding diminishing improvements in voltage at the expense of increased generator size and reduced mass. The predicted voltage output for the increased magnet was $165 \mathrm{mVpk}$ output compared to $64 \mathrm{mVpk}$ for the $1 \times 1 \times 1.5 \mathrm{~mm}^{3}$ size magnets (see figure 7). This is a factor of improvement of 2.6 in the open circuit voltages.

\subsection{Cantilever beam}

Despite being an excellent spring material for this application, the single-crystal silicon beams used in the Mk1 generator were found to be too brittle to handle during assembly. 


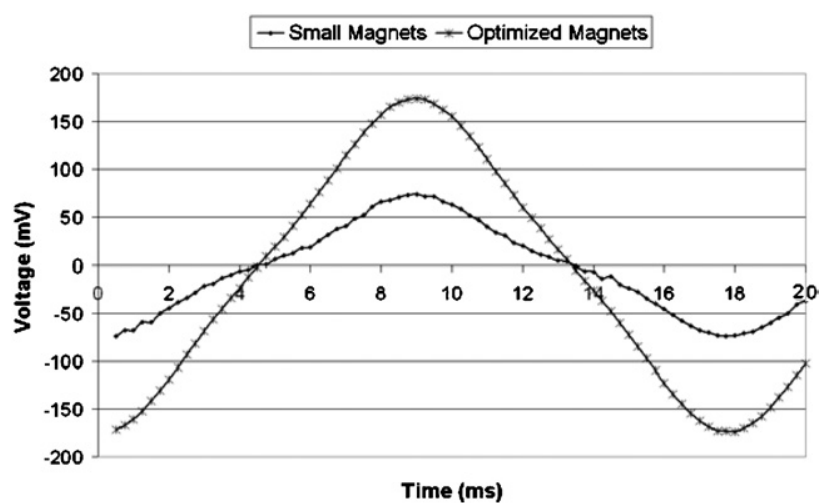

Figure 7. Simulated output voltages for optimized and small magnet generator configurations.

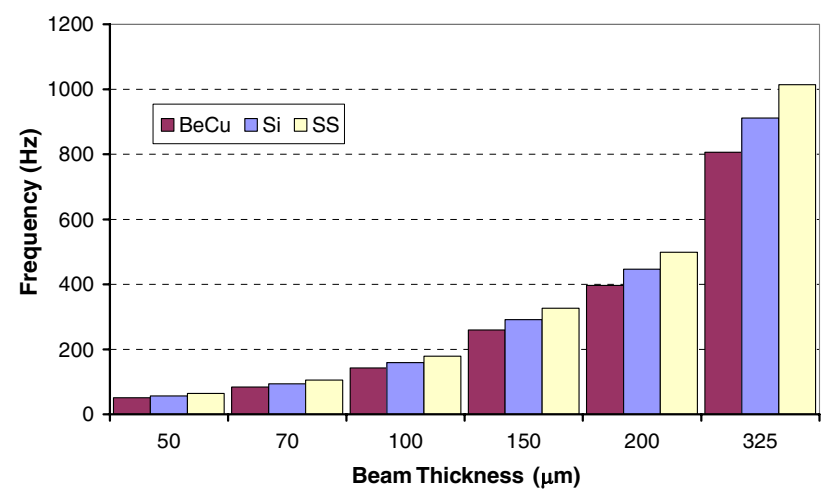

Figure 8. Generator frequency for varying beam thickness and material.

Therefore alternative metallic materials beryllium copper $(\mathrm{BeCu})$ and stainless steel type 302 full hard were investigated. These materials possess mechanical properties well suited to this application, in particular excellent fatigue characteristics. The metal beams have been fabricated by a combination of photolithography and spray etching. This involves coating both sides of the metal sheet with a UV sensitive photoresist and using contact lithography to define the beam shape. After exposure, the resist is developed leaving regions of the metal sheet exposed to a Ferric Chloride etchant which is sprayed simultaneously to both sides. This etches through the exposed metal leaving the desired beam geometry. This is a straightforward batch fabrication process enabling numerous structures to be fabricated simultaneously on each metal sheet.

The resonant frequency of the generator is defined by the beam geometry, material and the inertial mass. The resonant frequency of the generator versus beam thickness is shown in figure 8 for a magnet size of $2.5 \times 2 \times 1.5 \mathrm{~mm}^{3}$. These results were obtained from ANSYS modal analysis and demonstrate the range of frequencies attainable with standard sheet thicknesses. For this prototype $50 \mu \mathrm{m}$ thick $\mathrm{BeCu}$ was chosen which gives a predicted frequency of $51 \mathrm{~Hz}$.

\subsection{Coil properties}

In addition to the coil used in the Mk1 generator two further coils of identical dimensions were investigated. The three coils, denoted by A, B and C, were wound from 25,16 and
Table 1. Coil parameters.

\begin{tabular}{llrcl}
\hline Coil & $\begin{array}{l}\text { Wire diameter, } \\
\phi(\mu \mathrm{m})\end{array}$ & $\begin{array}{l}\text { No. of } \\
\text { turns }\end{array}$ & $R_{\text {coil }}(\Omega)$ & $\begin{array}{l}\text { Fill } \\
\text { factor }\end{array}$ \\
\hline A & 25 & 600 & 100 & 0.67 \\
B & 16 & 1200 & 400 & 0.45 \\
C & 12 & 2300 & 1500 & 0.53 \\
\hline
\end{tabular}

$12 \mu \mathrm{m}$ diameter enamelled copper wire respectively. Typical coil parameters are given in table 1 .

The length of wire used for each coil can be calculated from $L_{w}=R_{\text {coil }} A_{w} / \rho$ where $A_{w}$ is the cross sectional area of the wire and $\rho$ the resistivity of copper $\left(1.7 \times 10^{-8} \Omega \mathrm{m}\right)$. This gives wire lengths of $2.9,4.7$ and $10 \mathrm{~m}$ for coils A, B and $\mathrm{C}$ respectively. The coil fill factor, $F$, the ratio of the volume of conductor to the volume of the coil, is given by equation (11):

$$
F=\frac{L_{w} \phi^{2}}{4\left(R_{o}^{2}-R_{i}^{2}\right) t} .
$$

This gives coil fill factors of 0.67 for coil A, 0.45 for coil $\mathrm{B}$ and 0.53 for coil $\mathrm{C}$. This shows there is a difference in the density of the windings in each of the coils due to variations in the winding process. A higher fill factor is preferable since this indicates a higher number of turns within a given volume.

\section{Experimental analysis}

Testing of the generators was conducted using a shaker unit with accelerometer feedback and a programmable resistive load. The system is controlled by LabView software which allows the user to program long sequences of tests to automatically characterize each generator over a range of acceleration levels, load resistances and frequencies. Great care was taken to mount the accelerometer and generators concentrically on the shaker unit to ensure reliable and repeatable acceleration readings and results. The following results were taken at an acceleration level of $60 \mathrm{mg}$, unless otherwise noted.

\subsection{Evaluation of optimized magnets}

The first experiment compared the $1 \times 1 \times 1.5 \mathrm{~mm}^{3}$ magnets to the optimized dimensions, $2.5 \times 2 \times 1.5 \mathrm{~mm}^{3}$ using coil A. The comparison is shown in figure 9 which shows the measured voltage across a $9 \mathrm{M} \Omega$ load resistance versus frequency.

The observed resonant frequency of $56.6 \mathrm{~Hz}$ shows reasonable agreement with the FEA model being within $10 \%$ of the predicted result. The difference is due to the tolerance on the thickness of the beam and the nonlinear response of the generator. The peak output voltage increases from $39 \mathrm{mVrms}$ with the original magnets to $88 \mathrm{mVrms}$ with the optimized magnet configuration, an increase of $225 \%$. Next, the power output to the load was measured for the optimized magnet configuration. The optimum load resistance was determined by measuring the power output at resonance over a wide range of resistance values, the optimum being $150 \Omega$. The maximum power output of $17.8 \mu \mathrm{W}$ was obtained with a voltage output of $52 \mathrm{mVrms}$ across the optimum load as shown in figure 10 . Both figures 9 and 10 show evidence of the magnets on the beam touching the base at peak amplitude. The base was modified to avoid this in subsequent experiments. 


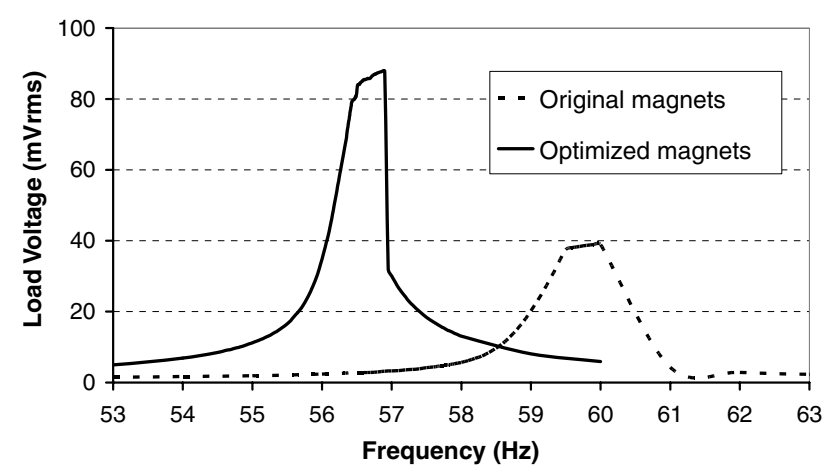

Figure 9. Generated output voltages for the small and optimized magnet configurations.

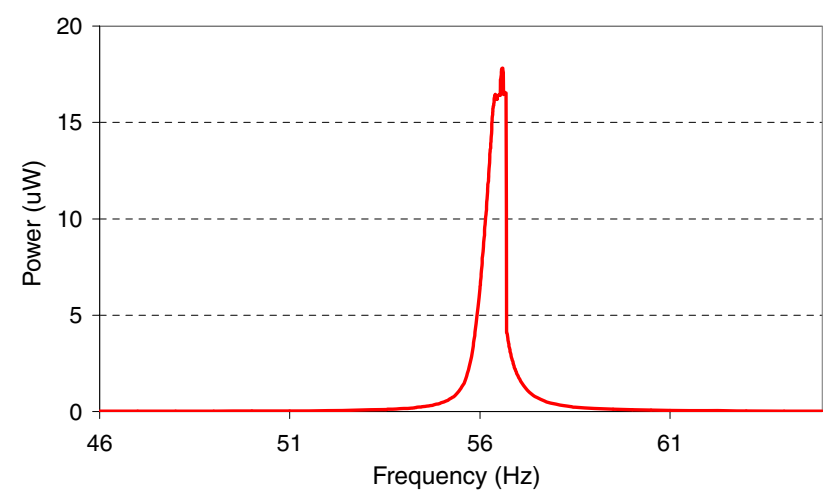

Figure 10. Optimum power output of the optimized magnet configuration generator.

Table 2. Generator results.

\begin{tabular}{llllr}
\hline Coil & $\begin{array}{l}\text { Resonant } \\
\text { frequency }\end{array}$ & $\begin{array}{l}\text { Load } \\
\text { resistance }\end{array}$ & $\begin{array}{l}\text { Power at } \\
\text { resonance }\end{array}$ & $\begin{array}{r}\text { Voltage at } \\
\text { resonance }\end{array}$ \\
\hline A & $52.1 \mathrm{~Hz}$ & $200 \Omega$ & $44.7 \mu \mathrm{Wrms}$ & $95 \mathrm{mVrms}$ \\
$\mathrm{B}$ & $51.64 \mathrm{~Hz}$ & $500 \Omega$ & $45.8 \mu \mathrm{Wrms}$ & $151 \mathrm{mVrms}$ \\
$\mathrm{C}$ & $53.2 \mathrm{~Hz}$ & $4 \mathrm{k} \Omega$ & $45.7 \mu \mathrm{Wrms}$ & $428 \mathrm{mVrms}$ \\
\hline
\end{tabular}

\subsection{Evaluation of coil types}

Coils of types A, B and C were each located on an individual generator base and the same beam assembly mounted on each base in turn. The resulting output voltage across the optimum load for each coil type is shown in figure 11. As expected, the output voltage increases with increasing number of turns with 95,151 and $428 \mathrm{mVrms}$ being generated from the 600 , 1200 and 2300 turn coils respectively. The generated power is very similar for each device as shown in figure 12 with the full set of results summarized in table 2 . The generated power is essentially the same for each device because the improved voltage output is offset by the increased coil resistance. This is reflected by the equation for damping factor (equation (10)).

The increase in voltage for the generator with coil $\mathrm{C}$ to over $400 \mathrm{mVrms}$ should be sufficient to enable conventional passive rectification and step up circuits to be implemented. Furthermore, the generator output power has increased to over $45 \mu \mathrm{W}$ at $60 \mathrm{mg}$ excitation. This is due to improvements in the assembly of the device, in particular the clamping and alignment of the beam, which leads to reduced energy parasitic damping and an increased open circuit $Q$-factor.

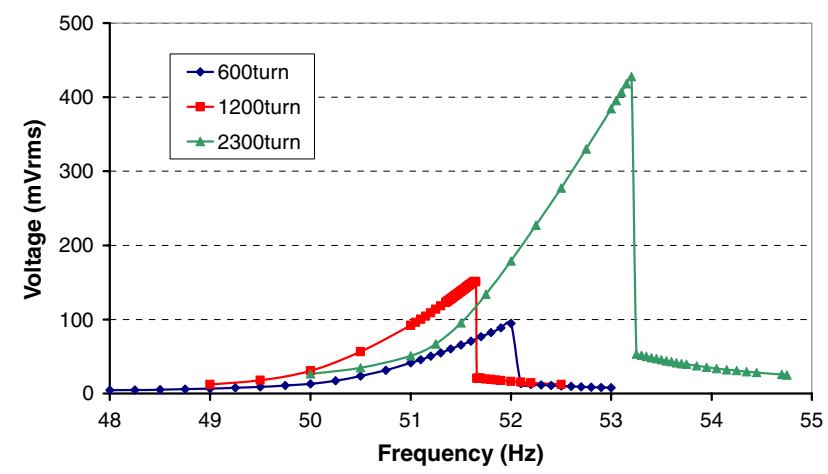

Figure 11. Output voltages for 600/1200/2300 turn coils across optimum load resistance.

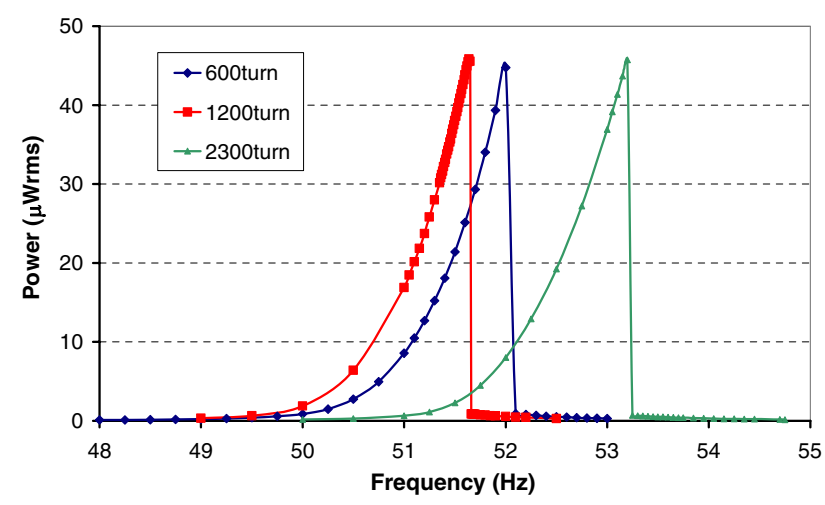

Figure 12. Output power for 600/1200/2300 turn coils with optimum load resistance.

The high $Q_{O / C}$ means these generators demonstrated nonlinear behaviour at very low acceleration levels $(<3 \mathrm{mg})$ and the $Q$-factor cannot be determined from a frequency amplitude plot such as that shown in figure 12. Therefore, the $Q$-factor was measured by observing the decay in the voltage signal from the generator. The coil $\mathrm{C}$ generator was initially driven at resonance at $20 \mathrm{mg}$ and the excitation stopped abruptly. Figure 13(a) shows the decay in the generator open circuit output over $6.5 \mathrm{~s}$, figure 13(b) shows the decay immediately after turning off the excitation and figure 13(c) shows the signal approximately $1.5 \mathrm{~s}$ later. $Q$-factor can be calculated from equation (12) where $f_{0}$ is the frequency, $V_{1}$ and $V_{2}$ are the voltage amplitudes at a time interval $\Delta t$ apart:

$$
Q=\frac{\pi f_{0} \Delta t}{\ln \left(\frac{V_{1}}{V_{2}}\right)} .
$$

The $Q$-factor calculated from figure $13(b)$ is 520 whilst the $Q$-factor in (c) is 274 . This indicates that the parasitic damping is a function of amplitude and decreases with increasing excitation acceleration levels. This behaviour could be due to the magnets extending beyond the influence of the coil at the higher amplitudes which, even when open circuit, produces a damping effect.

\section{Theoretical analysis of device performance}

Given the nonlinear behaviour of the high- $Q$ generators, the generator used to evaluate the optimum magnet size (described 

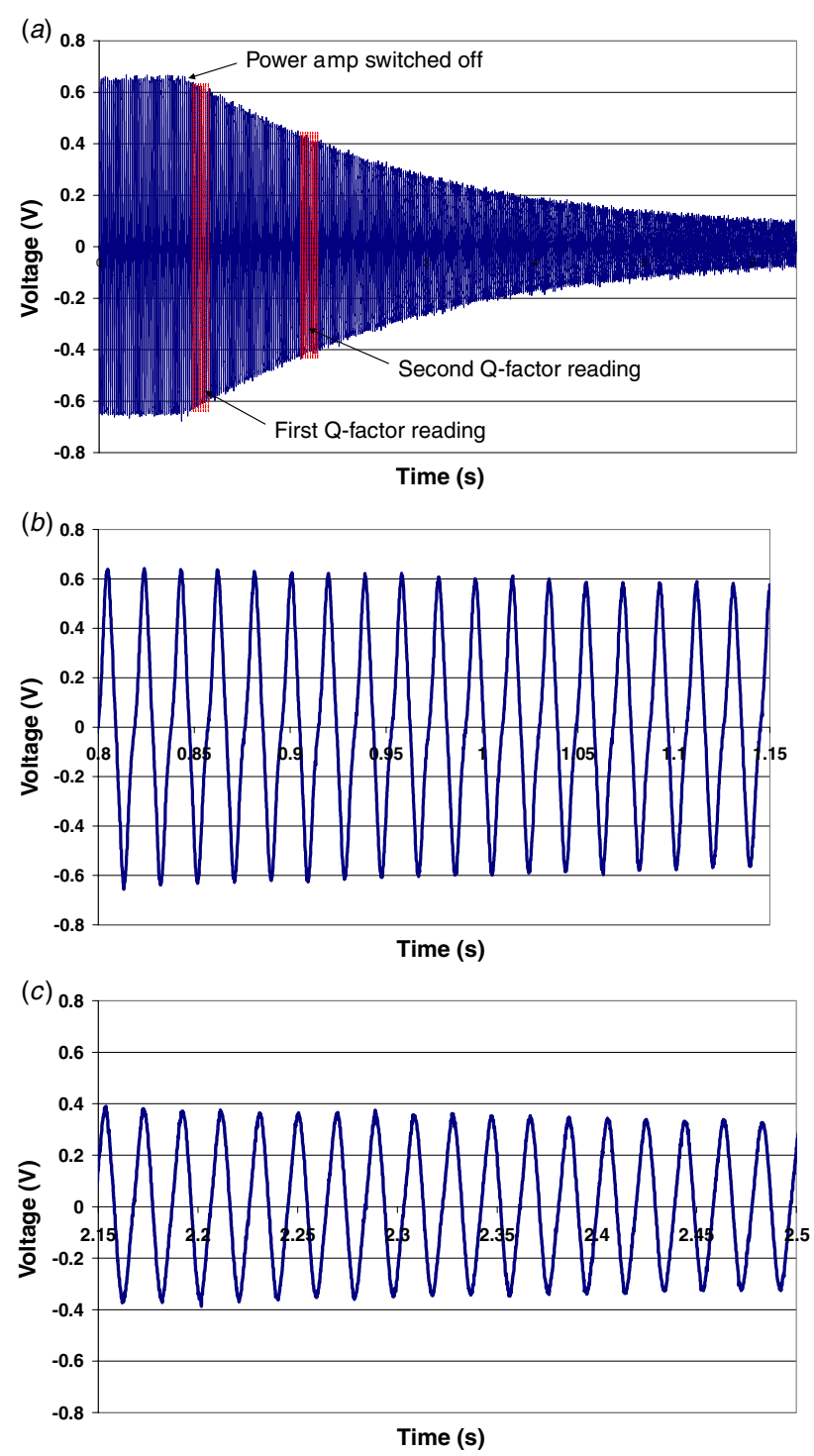

Figure 13. Decay plots from generator with coil C $(a)$ over $6.5 \mathrm{~s}$ (top), (b) initial decay after switching shaker off, $Q=520$ (middle), (c) decay $1.5 \mathrm{~s}$ later, $Q=274$ (bottom).

in section 6.1 , peak power $17.8 \mu \mathrm{W}$ at $56.6 \mathrm{~Hz}$ ) was compared with the theory. This device was more highly damped and did not demonstrate nonlinear frequency or nonlinear damping behaviour below $0.29 \mathrm{~m} \mathrm{~s}^{-2}$. The generator was compared with the theoretical calculations for power supplied to the generator from the environment (equation (2)), the maximum power delivered to the electrical domain (equation (8)) and the power delivered to the load (equation (9)).

Firstly, the closed and open loop quality factors were measured at an acceleration level below the onset of nonlinear behaviour. The measured values of $Q_{T}$ and $Q_{\mathrm{OC}}$ at $20 \mathrm{mg}$ were 119 and 232 respectively and from equation (5), $Q_{E}$ equals 243. It can be seen that the optimum damping conditions have very nearly been met and it is reasonable to use equations (8) and (9). The coil and load resistances, $R_{\text {Coil }}$ and $R_{\text {Load }}$ used in equation (9) were equal to 100 and $200 \Omega$ respectively.

The predicted power outputs from the theoretical equations and the measured output from the generator

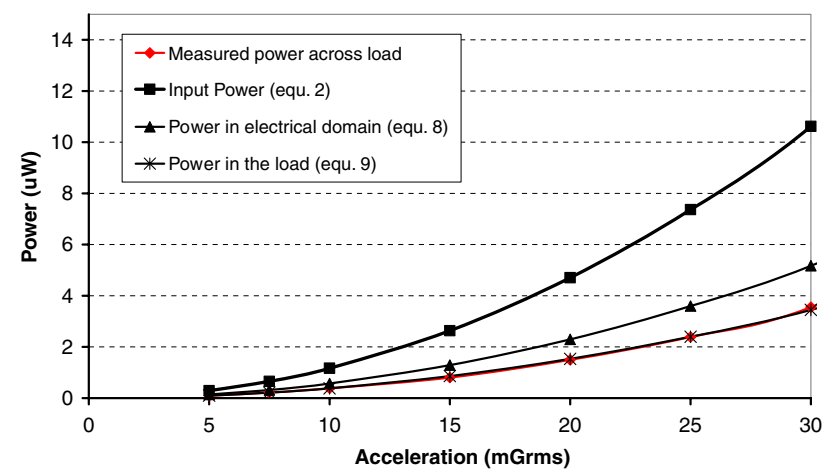

Figure 14. Comparison of theoretical and measured power output.

have been plotted versus acceleration up to $0.29 \mathrm{~m} \mathrm{~s}^{-2}$ in figure 14. This shows excellent agreement between measured and predicted power levels and demonstrates that the cantilever microgenerator is converting $30 \%$ of the total power dissipated in the generator to electrical power delivered to the load. Due to the relative coil and load resistances, one third of the power converted into the electrical domain is lost within the coil. Equation (9) highlights the importance of reducing coil resistance and increasing load resistance as long as the optimum damping condition is maintained. The theoretical and practical results show excellent agreement up to $0.29 \mathrm{~m} \mathrm{~s}^{-2}$. Beyond this point, the nonlinear behaviour alters the frequency response and parasitic damping levels and determining accurate values for the theoretical analysis is not straightforward. Work to analyse the theoretical response of the high- $Q$ generators in the nonlinear region is ongoing.

\section{A comparison of selected energy harvesting devices}

Comparing different vibration energy harvesters is not straightforward since the amount of data presented in published works varies considerably. Therefore, inevitably some factors have to be extrapolated from the data given and any comparison should only be treated as a guide. Mitcheson et al [12] presented a comparison where they estimated the relative input powers and calculated an approximate efficiency. In this paper, we have derived a figure for normalized power density (NPD) which is simply the stated power output of the device normalized to acceleration level and divided by the volume. Frequency is not considered since resonant generators are fixed in frequency whereas acceleration levels applied during testing can be varied. Neither the efficiency nor NPD metric is ideal since they both ignore important factors such as bandwidth but unfortunately insufficient data exist in the literature to enable this to be included. The Perpetuum generator [14], for example, has a significantly broader bandwidth than the generator presented here enabling it to harvest energy from a wider range of frequencies. Nonetheless, the comparison of NPD for different devices can provide an indication of relative performance levels and provide a useful insight into trends and advances.

Since power output varies with acceleration ${ }^{2}$, the calculated NPD is given by $P / A^{2} V$ where $P$ is the stated power 


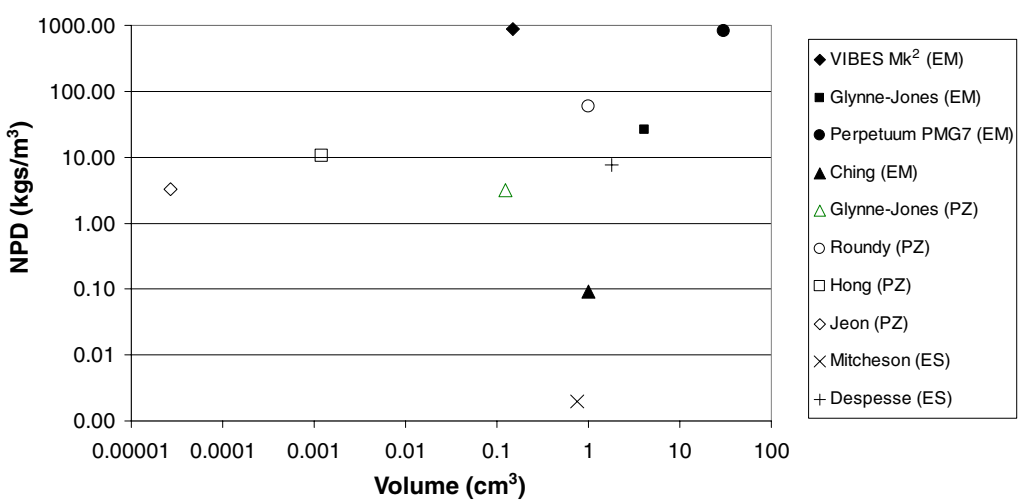

Figure 15. Normalized power density versus device volume.

Table 3. Comparison of generators.

\begin{tabular}{|c|c|c|c|c|c|c|}
\hline Generator $^{\mathrm{a}}$ & Freq $(\mathrm{Hz})$ & Acceln $\left(\mathrm{m} \mathrm{s}^{-2}\right)$ & Inertial mass $(\mathrm{g})$ & Volume $\left(\mathrm{cm}^{3}\right)$ & Power $(\mu \mathrm{W})$ & $\mathrm{NPD}\left(\mathrm{kgs} \mathrm{m}^{-3}\right)$ \\
\hline VIBES Mk2 EM & 52 & 0.589 & 0.66 & 0.15 & 46 & 883.97 \\
\hline Glynne-Jones [13] EM & 99 & 6.85 & 2.96 & 4.08 & 4990 & 26.07 \\
\hline Perpetuum [14] EM & 100 & 0.400 & 50 & 30 & 4000 & 833.33 \\
\hline Ching [15] EM & 110 & 95.5 & 0.192 & 1 & 830 & 0.09 \\
\hline White [16] PZ & 80 & 2.3 & 0.8 & 0.125 & 2.1 & 3.18 \\
\hline Roundy [17] PZ & 120 & 2.5 & 9.15 & 1 & 375 & 60.00 \\
\hline Hong [18] PZ & 190 & 71.3 & 0.01 & 0.0012 & 65 & 10.67 \\
\hline Jeon [19] PZ & 13900 & 106.8 & $2.20 \times 10^{-07}$ & 0.000027 & 1 & 3.25 \\
\hline Mitcheson [20] ES & 30 & 50 & 0.1 & 0.75 & 3.7 & 0.002 \\
\hline Despesse [21] ES & 50 & 8.8 & 104 & 1.8 & 1052 & 7.55 \\
\hline
\end{tabular}

${ }^{a}$ Generators are labelled by technology: EM, electromagnetic; PZ, piezoelectric; ES, electrostatic.

output and $V$ is the reported volume of the generator. The selection and details of energy harvesters from the literature used in the comparison are detailed in table 3 and a comparison is shown diagrammatically in figure 15 .

\section{Conclusions}

This paper has presented a small (components volume $0.1 \mathrm{~cm}^{3}$, practical volume $0.15 \mathrm{~cm}^{3}$ ) electromagnetic vibration energy harvesting device optimized for a low level of ambient vibration based upon real application data. It is capable of producing useful power from a vibration level of $60 \mathrm{mg}$, delivering $46 \mu \mathrm{W}$ to a resistive load of $4 \mathrm{k} \Omega$ when the device is shaken at its resonant frequency of $52 \mathrm{~Hz}$. This is a power density of $307 \mu \mathrm{W} \mathrm{m}^{-3}$. The generator delivers $30 \%$ of the total power dissipated in the generator to electrical power in the load. This generator compares very favourably with other demonstrated examples in the literature, both in terms of normalized power density and efficiency.

From the basic equations governing electromagnetic generators it is clear that the generated energy decreases with device volume, and reducing input vibration acceleration ${ }^{2}$. With the exception of the Perpetuum generator [14] (which is a much larger generator, $30 \mathrm{~cm}^{3}$ ), all the existing generators (both piezoelectric and electromagnetic) described in the literature [3] produce optimum power densities at input vibrations greater than $2 \mathrm{~m} \mathrm{~s}^{-2}$. One of the aims of this study was to demonstrate that it was possible for a device of this volume to produce useful power from only $0.59 \mathrm{~m} \mathrm{~s}^{-2}$ of ambient vibration.
To demonstrate that the power from the device is 'useful' (i.e. of a voltage level and source impedance that permits rectification and storage), a prototype rectification and multiplier circuit has been fitted to the generator (in a manner similar to that described by Ching et al [15]), and is capable of charging a capacitor to power the transmission of a periodic radio signal. This circuitry was not optimized, and we continue to work on a demonstrator that will better show the potential of a miniature wireless sensor node based on this generator.

\section{Acknowledgments}

The authors would like to acknowledge the financial support of the European Union who funded this research through the Framework 6 Programme. We would like to acknowledge Dr Steve Roberts at Perpetuum Ltd for his assistance in setting up the vibration test rig.

\section{References}

[1] Banazwski B and Shah R K 2003 The role of fuel cells for consumer electronic products and toys Proc. Ist Int. Conf. on Fuel Cell Science, Engineering and Technology (Rochester, NY) pp 149-55

[2] Epstein A H 2004 Millimeter-scale, micro-electro-mechanical systems gas turbine engines J. Eng. Gas Turbines Power 126 205-26

[3] Beeby S P, Tudor M J and White N M 2006 Energy harvesting vibration sources for microsystems applications Meas. Sci. Technol. 17 R175-R195 
[4] Roundy S, Wright P K and Rabaye J 2003 A study of low level vibrations as a power source for wireless sensor nodes Comput. Commun. 26 1131-44

[5] Starner T and Paradiso J A 2004 Human generated power for mobile electronics Low Power Electronics Design ed C Piguet (Boca Raton, FL: CRC Press)

[6] von Büren T, Lukowicz P and Tröster G 2003 Kinetic energy powered computing - an experimental feasibility study Proc. 7th IEEE Int. Symp. on Wearable Computers ISWC '03 (White Plains, NY) pp 22-4

[7] El-Hami M et al 2001 Design and fabrication of a new vibration-based electromechanical power generator Sensors Actuators A 92 335-42

[8] Williams C B, Shearwood Harradine M A, Mellor P H, Birch T S and Yates R B 2001 Development of an electromagnetic micro-generator IEE Proc. Circuits Devices Syst.148 337-42

[9] Stephen N G 2006 On energy harvesting from ambient vibration J. Sound Vib. 293 409-25

[10] Glynne-Jones P, Tudor M J, Beeby S P and White N M 2004 An electromagnetic, vibration-powered generator for intelligent sensor systems Sensors Actuators A $110344-49$

[11] O'Donnell T, Saha C, Beeby S and Tudor J 2006 Scaling effects for electromagnetic vibrational power generators Proc. Design, Test, Integration and Packaging of MEMS and MOEMS (Stresa, Italy, 26-28 April 2006) Proc. SPIE 4019 279-85

[12] Mitcheson P D, Reilly E K, Wright P K and Yeatman E M 2006 Transduction mechanisms and power density for MEMS inertial energy scavengers Proc. Power MEMS 06 (Berkeley: USA) pp 275-78
[13] Glynne-Jones P 2001 Vibration powered generators for self-powered microsystems PhD Dissertation Department of Electronics and Computer Science, University of Southampton, UK

[14] PMG7 data sheet, Perpetuum Ltd. www.perpetuum.co.uk.

[15] Ching N N H, Wong H Y, Li W J, Leong P H W and Wen Z 2002 A laser-micromachined vibrational to electrical power transducer for wireless sensing systems Sensors Actuators A 97-98 685-90

[16] Glynne-Jones P, Beeby S P and White N M 2001 Towards a piezoelectric vibration powered microgenerator IEE Proc. Sci. Meas. Technol. 148 68-72

[17] Roundy S and Wright P K 2004 A piezoelectric vibration based generator for wireless electronics Smart Mater. Struct. 13 1131-42

[18] Hong Y K and Moon K S 2005 Single crystal piezoelectric transducers to harvest vibration energy Proc. SPIE 6048 60480E-1

[19] Jeon Y B, Sood R, Jeong J-h and Kim S G 2005 MEMS power generator with transverse mode thin film PZT Sensors Actuators A 122 16-22

[20] Mitcheson P, Stark B, Miao P, Yeatman E, Holmes A and Green T 2003 Analysis and optimisation of MEMS on-chip power supply for self powering of slow moving sensors Proc. Eurosensors XVII (Guimaraes: Portugal) pp 30-1

[21] Despesse G et al 2005 Fabrication and characterisation of high damping electrostatic micro devices for vibration energy scavenging Proc. Design, Test, Integration and Packaging of MEMS and MOEMS pp 386-90 